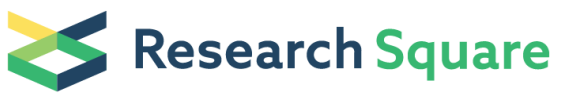

Preprints are preliminary reports that have not undergone peer review.

They should not be considered conclusive, used to inform clinical practice, or referenced by the media as validated information.

\title{
A Comparative Evaluation of Anti-Inflammatory and Arthritic Effects of bradykinin inhibitor Zaltoprofen over Piroxicam in Murine models: A randomized experimental study.
}

Balakrishnan Sadasivam.

AllMS: All India Institute of Medical Sciences

SANTENNA Chenchula ( $\nabla$ csanten7@gmail.com )

All India Institute of Medical Science - Bhopal https://orcid.org/0000-0001-7466-1037

Sunil kumar

AllMS: All India Institute of Medical Sciences

Ratinder Jhaj

AllMS: All India Institute of Medical Sciences

Saman Pathan

AllMS: All India Institute of Medical Sciences

Research Article

Keywords: Inflammation, arthritis, Carrageenan, Formalin, Zaltoprofen, Piroxicam

Posted Date: February 14th, 2022

DOI: https://doi.org/10.21203/rs.3.rs-1353934/v1

License: (c) (1) This work is licensed under a Creative Commons Attribution 4.0 International License. Read Full License 


\section{Abstract}

Background and Aims: Inflammation is an essential biochemical protective response of vascularized tissues, broadly classified into acute and chronic inflammation. Zaltoprofen, a unique propionic acid group of NSAID, acts by blocking the augmenting effect of bradykinin. The present study was conducted to evaluate the acute and chronic anti-inflammatory (arthritic) potential of Zaltoprofen over piroxicam in murine models.

\section{Methods:}

A total of 24 healthy Wister rats (200-250 g) of either sex (24 in each model) were obtained from the central animal house, AllMS, Bhopal and categorized into four different groups, six in each. Zaltoprofen was used as a test and Piroxicam as a Standard (Per Os) agent. The anti-inflammatory or arthritic potential was evaluated and compared by acute and chronic inflammation (carrageenan and formalin-induced)models.

\section{Results:}

In the acute inflammation model, Zaltoprofen when compared to Negative control $(\mathrm{NaCl} 10 \mathrm{ml} / \mathrm{kg})$ there was a significant inhibition in paw volume $(\mathrm{P}<0.001)$ at different time scales with two different doses of Test compound (Zaltoprofen 10\&20mg/kg). However, in the chronic inflammation model, Zaltoprofen 10 $\mathrm{mg} / \mathrm{kg} \& 20 \mathrm{mg} / \mathrm{kg}$ doses of the Test compound had been shown a significant reduction in the chronic inflammation comparable to the negative control (NaCl $10 \mathrm{ml} / \mathrm{kg}$ ), though the efficacy was lesser than Positive control (Piroxicam 10mg/kg) $(P \leq 0.05)$.

\section{Conclusions:}

Zaltoprofen possesses significant acute and chronic anti-inflammatory and arthritic effects by inhibiting various inflammatory mediators. Therefore, Zaltoprofen can be explored as a potential alternative agent for the treatment of acute inflammatory and chronic inflammatory or arthritis conditions in human beings.

\section{Introduction}

Inflammation is an essential biochemical protective response of vascularized tissues that delivers leukocytes and molecules of host defence from the circulation to the sites of infection and cell damage to eliminate the offending agents [1]. It serves to clear the host of both the initial cause of cell injuries like microorganisms and toxins and the consequences of such injury, which may be necrosis of cells and tissues. Various mediators of defence include phagocytic leukocytes, antibodies, and complement proteins. During the inflammation, circulating inflammatory cells and proteins reach the injured tissues and activate the recruited and resident cells, as well as the soluble molecules, which could help to get rid of the harmful or unwanted substances. Inflammation is essential for survival to overcome the infections and healing of wounds

Acute inflammation develops within some minutes of tissue injury or infection, having characteristics of fluid and plasma proteins exudation, leading to oedema, and the migration of leukocytes, primarily neutrophils, and is very short in duration. However, if the offending stimulus is removed, the reaction subsides, and residual injury is repaired. Rheumatoid arthritis (RA) is a type of chronic inflammation, which is longer in duration and is associated with more tissue destruction and scarring [1]. Both Steroidal and nonsteroidal anti-inflammatory drugs (NSAIDs) are the most commonly used drugs for the prevention and treatment of inflammation. However, on long term use, NSAIDs are associated with gastrointestinal, hepatic, renal and cardiac side effects, etc. [2]. Hence, it is very essential to find drugs with lower side effects for the treatment of inflammatory diseases.

Zaltoprofen is a unique NSAID from the propionic acid group with a tri-cyclic formula. Its chemical name is 2-(6-oxo-5Hbenzo[b][1]benzothiepin-3-yl) propanoic acid[3]. It has a potent analgesic and antipyretic activity [4-6]. It acts by blocking the, augmenting effect of bradykinin a potent endogenous algesic or sensitizing substance, acts through Bradykinin-1(inducible) and Bradykinin-2(constitutive) receptors. Zaltoprofen acts by inhibiting Gq protein-coupled B2 receptor-mediated action on a-amino-3-hydroxy-5-methyl-4-isoxazole propionic acid receptor (AMPA) currents, through the inhibition of protein kinase-C activation in the primary sensory neurons [7]. Additionally, several different signal transduction pathways, including phospholipase $C$ (PLC), phospholipase $A_{2}$, lipooxygenase (LOX), cyclooxygenase (COX) enzymes in dorsal root ganglion (DRG) cells [7]. Zaltoprofen also exhibits an anti-inflammatory effect by inhibiting the cyclooxygenase-2(COX2) enzyme selectively and inhibits prostaglandin-E2 production at inflammatory sites [6-8]. Findings of an in vivo study by Tang et al. has shown that Zaltoprofen inhibits bradykinin-induced 12-lipoxygenase (LOX) activation in vitro and the slow bradykinin-induced onset of substance P release from dorsal root ganglion (DRG) neurons [9]. A study by Matsumoto et al. concluded that Zaltoprofen has inhibitory action specifically on bradykinin-induced nociception in nociceptor endings through B2-type bradykinin receptors [10]. An experimental study finding by Kameyama et.al, has shown that zaltoprofen inhibits bradykinin-induced nociceptive responses more significantly, with a better safety margin, having less potential to induce gastric ulcers than indomethacin [6]. Santenna $\mathrm{C}$ et al. concluded that zaltoprofen analgesic efficacy was non-inferior to a standard NSAID piroxicam in experimental murine models of acute pain [5]. A multicentric, double-blind, double-dummy, randomized, parallel-group comparative study by Pareek et.al concluded that clinically zaltoprofen is non-inferior to Diclofenac [11]. Akihico T et al., had shown that zaltoprofen might be a potential agent to act against malignant phenotypes in chondrosarcomas via activation of PPAR $y$ and inhibition of MMP 2 activity [12]. A randomized placebo-controlled, double-blind, phase II trial by Akihico T et al., had found that Zaltoprofen can be a potential newer therapeutic agent, to stabilize disease progression for patients with diffuse tenosynovial giant cell tumour (D-TGCT) or unresectable L-TGCT [13]. A study by Okamoto et al., had reported that Zaltoprofen improves the loss of body weight in both the Con Ainduced mouse and carbon tetrachloride-induced rat sickness behaviour models, like other commonly used NSAIDs Aspirin and Indomethacin [14][Figure 1].

Zaltoprofen has shown its anti-inflammatory action at $\mathrm{ED}_{50}, 1-5 \mathrm{mg} / \mathrm{kg}$, per oral [4]. Hence we have planned to study the dose range above $5 \mathrm{mg} / \mathrm{kg}$ body weight. In addition, till now there were no studies on the anti-inflammatory activity comparitive efficacy of zaltoprofen over the very commonly prescribing antiinflammatory agent piroxicam. Therefore, considering this background, we have evaluated and compared the anti-inflammatory and arthritic activity of Zaltoprofen efficacy over piroxicam in the Murine models of acute and chronic inflammation or arthritis.

Page 2/8 


\section{Methodology Experimental Animals:}

Total 48 healthy male Wister albino rats $(200-250 \mathrm{~g})$ were used for the experimentations which were obtained from the Central Animal House of the institution, All India Institute of Medical Sciences (AlIMS) Bhopal, India. All the animals were adapted to the new environment minimum of two weeks before initiating the experiments using six animals in each group. The animal house temperature was maintained at $25^{\circ} \mathrm{C} \pm 2{ }^{\circ} \mathrm{C}$ with 12 hours of light and 12 hours day cycle and they were allowed to have free access to standard pellet diet and water. The present experimental study was carried out as per the Committee for the purpose of control and supervision of experiments on animals(CPCSEA) guidelines after the approval by the Institutional Animal Ethics Committee (IAEC), AlIMS Bhopal, India (AIIMS/BHOPAL/IAEC/2017/03 Date: $10^{\text {th }}$ August 2017).

\section{Chemicals and drugs:}

Chemicals Carrageenan and Formalin were obtained from Himedia Laboratories Pvt. Ltd (Mumbai, India). The Test compound Zaltoprofen was obtained from the JB Chemicals and Pharmaceuticals Ltd (Mumbai, India) and the standard agent Piroxicam from the Medipol Pharmaceutical India Pvt. Ltd. (New Delhi, India).

\section{Experimental Dosing:}

All the 24 experimental rats were randomly divided into four different groups ( $n=6$ in each group) in each model[ Figure 2].

Group I (Negative Control) were treated orally with normal saline (NS) $10 \mathrm{ml} / \mathrm{kg}$ body weight.

Group II (Positive Control) were treated orally with piroxicam $10 \mathrm{mg} / \mathrm{kg}$ body weight (PRC10).

Group III (Test-I) were treated orally with Zaltoprofen $10 \mathrm{mg} / \mathrm{kg}$ body weight (ZLT10).

Group IV (Test-II) were treated orally with Zaltoprofen $20 \mathrm{mg} / \mathrm{kg}$ body weight (ZLT20).

Experimental induction of acute and chronic inflammation:

\section{Carrageenan-Induced Acute Inflammation:}

Carrageenan induced paw oedema is the unique model of acute inflammation having large reproducibility. Inflammation was induced in rats according to previous standard research protocols [15]. Six animals of either sex in each group were used in the total four groups. Rats were administered orally $0.9 \% \mathrm{NaCl}$ $(10 \mathrm{ml} / \mathrm{kg})$, zaltoprofen $(10 \mathrm{mg} / \mathrm{kg}$ and $20 \mathrm{mg} / \mathrm{kg})$, and piroxicam $(10 \mathrm{mg} / \mathrm{kg})$ dissolved in $0.9 \%$ normal saline, $1 \mathrm{~h}$ before carrageenan administration. After that

$0.1 \mathrm{ml}$ of $1 \%$ carrageenan dissolved in normal saline was injected subcutaneously into the right hind paw for the induction of acute inflammation. The average volume of the left hind paw of each mouse was measured before injecting carrageenan, and at 30 minutes, 1 hour, 2 hours, and 3 hours after injection of carrageenan, using a digital plethysmometer (ORCHID Scientific \& Innovative India, Pvt. Ltd. India)[15]. By using the following formula we have calculated the percentage of inhibition in comparison with the control group.

$\%$ Inhibition $=[($ Volume of oedema $($ control $)-$ Volume of oedema (test) $] /$ Volume of oedema $($ control $) \times 100$.

\section{Formalin-induced arthritis:}

In the formalin-induced chronic inflammation model, paw oedema was induced by subaponeurotic administration of $0.1 \mathrm{ml}$ of $2 \%$ formaldehyde 30 minutes after the oral administration of standard /test/vehicle compounds on day 1 and day 3 respectively. Experimental animals were administered daily, with test drug Zaltoprofen $(10 \mathrm{mg} / \mathrm{kg}$ and $20 \mathrm{mg} / \mathrm{kg})$ and standard piroxicam $(10 \mathrm{mg} / \mathrm{kg}$ ) orally for 10 days. The daily changes in paw oedema or linear crosssection (LCS) of each rat, below the ankle joint, was measured daily with a digital vernier calliper (1). The difference in the paw volume on day 1 and day 10 was considered as inflammatory oedema. Volume changes in Test I, Test II and standard groups were compared with that of control and percentage inhibition was calculated.

The percentage inhibition of the inflammation was determined by the formula;

$\%$ Inhibition $=1-(\mathrm{dt} / \mathrm{d} c) \times 100$.

Statistical procedure: Data were analyzed using SPSS version 22.0. Results were expressed as mean \pm SD $(n=6)$. The percentage inhibition of the inflammation was calculated for each model. Comparison between all the four different groups was done by using one-way ANOVA followed by Bonferroni's test to compare the difference between groups at different time intervals. P-value less than 0.005 was considered statistically significant when compared with control $\left({ }^{*} \mathrm{p}<0.005\right)$.

\section{Results}

Acute anti-inflammatory activity: 
The mean of the basal paw oedema volume was comparable in all the groups (Table.1). After 60 and 120 minutes, the mean decrease in paw volume in all three groups (ZLT10mg/kg, ZLT $20 \mathrm{mg} / \mathrm{kg} \& \mathrm{PRC} 10 \mathrm{mg} / \mathrm{kg}$ ) was statistically significant when compared to the negative control group (P< 0.001$)$. For the negative control group, the injection of carrageenan caused localized oedema, after $30 \mathrm{~min}$. The swelling increased progressively from $30 \mathrm{~min}$ onwards. $\mathrm{A}$ maximum volume of the paw was observed in animals of negative control at $120 \mathrm{~min}(2.31 \pm 0.348)(0.289 \pm 0.008 \mathrm{ml})$ after injecting carrageenan. Test- $\mathrm{I}$ (zaltoprofen $10 \mathrm{mg} / \mathrm{kg}$ ) and Test-II (zaltoprofen $20 \mathrm{mg} / \mathrm{kg}$ ) caused significant decrease in the paw volume $(* \star * \mathrm{P}=<0.001)$ compared to control $(1.43 \pm 0.068$ and $1.36 \pm 0.128$ ) with $38.24 \%$ and $40 \%$ inhibition of inflammation respectively [Table I and Figure 3]. The standard drug (piroxicam20mg/kg) significantly reduced $\left({ }^{\star \star *} \mathrm{P}<0.001\right)$ the paw volume $(1.28 \pm 0.088)$ with $44.52 \%$ inhibition of inflammation. Percentage inhibition of acute inflammation was greater in the standard drug piroxicam group (Positive Control) compared to Test groups I\&II (ZLT10mg/kg and ZLT20mg/kg), at all-time intervals. Similarly, the percentage inhibition in the Test-II (ZLT20mg/kg) group was greater than that of Test-I (zaltoprofen 10mg/kg) at all-time intervals [Table II and Figure 4].

\section{Chronic anti-inflammatory (Arthritic) activity:}

Administration of formalin caused an increase in paw thickness in all groups. In the case of negative control, the highest increase in paw thickness was observed on day 3 and slowly reduced, but not restored to its original paw thickness which was on day 1 . Table.III and IV depict the mean \pm SD difference in paw volume (LCS) calculated from day 1 to day 10, for each group. Lower the difference in the increase in paw volume (LCS), higher is the anti-inflammatory action. The mean difference in LCS in all the three drug-treated groups was significantly less when compared to Negative Control $(P<0.001)$, though the mean difference in LCS was higher in Test I and II (zaltoprofen 10 and $20 \mathrm{mg} / \mathrm{kg}$ ) than that in the piroxicam group (Positive Control). The lowest difference in LCS was found in the Positive Control (piroxicam $10 \mathrm{mg} / \mathrm{kg}$ ) group. Formalin-induced paw oedema was reduced significantly $(p<0.001)$ with the administration of Test I and Test II (zaltoprofen $10 \mathrm{mg}$ and $20 \mathrm{mg}$ ), which was found to be a time-dependent and highest reduction in paw thickness or arthritis was observed on day 7 and (zaltoprofen $10 \mathrm{mg} / \mathrm{kg}$ ) Day 5 (Zaltoprofen $20 \mathrm{mg} / \mathrm{kg}$ ) respectively[Figure 5]. Percentage inhibition of chronic inflammation was higher in the piroxicam group (35.60\%) when compared to Test-I(zaltoprofen $10 \mathrm{mg} / \mathrm{kg})(18.90 \%)$ and Test-II (zaltoprofen20mg/kg) $(27.76 \%)$ at all-time intervals. Similarly, the percentage inhibition in Test-II (zaltoprofen20 mg/ $\mathrm{kg}$ ) group was higher than that of Test -I (zaltoprofen 10mg/kg) at all-time intervals [Table IV].

\section{Discussion}

The present experimental study has compared acute and chronic anti-inflammatory or arthritic potential of Zaltoprofen, using well-established experimental murine models. The Test drug Zaltoprofen was investigated and compared over piroxicam by using two different doses of Test compound(ZLT 10 and 20 $\mathrm{mg} / \mathrm{kg}$ vs. PRC10 mg/kg) in carrageenan-induced acute inflammation and formalin-induced chronic inflammation or arthritis models, and the results were depicted (Tables 1-3; Figures 1-3).

Carrageenan induced acute inflammation is a standard experimental model for acute inflammation. A major advantage of carrageenan is, single oral doses of drugs at non-toxic levels are effective; and it is similar to the early exudative stage of inflammation in humans and the model is linked with the activation of various inflammatory autacoids like histamine, serotonin, bradykinin, prostaglandins and leukotrienes. After injecting the carrageenan into the hind paw it induces progressive oedema that reaches its maximum within 3 hours [15]. Both the test doses of zaltoprofen showed significant anti-inflammatory activity in comparison to the negative control $(\mathrm{NaCl} 10 \mathrm{ml} / \mathrm{kg})$. However, when compared to a positive control (Piroxicam $10 \mathrm{mg} / \mathrm{kg}$ ) anti-inflammatory activity of test doses (TEST I \& II) of zaltoprofen were less than standard piroxicam. Inhibition of carrageenan-induced oedema by zaltoprofen can be attributed to its ability to inhibit the various mediators of inflammation like kinins and prostaglandins.

Formalin-induced chronic inflammation is a model for arthritis and is considered the most suitable experimental animal model which closely resembles arthritis in human beings. It is associated with the proliferative phase of chronic inflammation in human beings and is biphasic, an early neurogenic phage is mediated by substance-P and bradykinin followed by a later tissue mediated response where, histamine, 5-hydroxytryptamine (5-HT), prostaglandins (PGs) and bradykinin are known to be involved [16]. Zaltoprofen has shown a significant inhibition in paw volume in the formalin-induced chronic inflammation or arthritis model. Test-I (Zaltoprofen $10 \mathrm{mg} / \mathrm{kg}$ ) inhibited $19 \%$ of paw oedema, Test-II (zaltoprofen $20 \mathrm{mg} / \mathrm{kg}$ ) inhibited $28 \%$ of paw oedema, and standard drug piroxicam decreased $36 \%$ paw oedema respectively. Both the test doses showed significant anti-arthritic activity, though it was less than positive control piroxicam $10 \mathrm{mg} / \mathrm{kg}$. Therefore, as this model is more suitable and very commonly used to evaluate anti-inflammatory agents with probable anti-proliferative activity, the inhibitory effect of zaltoprofen on formalin-induced chronic inflammation, indicates its significant anti-proliferative and arthritic activity [17].

\section{Conclusion}

In conclusion, zaltoprofen in a dose-dependent manner is an effective agent in attenuating carrageen and formalin-induced acute and chronic inflammation and arthritis cascade in experimental murine models of inflammation or arthritis. Therefore, it can be explored and used as a potential therapeutic agent for acute and chronic inflammatory clinical conditions in humans, particularly those associated with arthritis.

\section{Declarations}

\section{Acknowledgements:}

The authors are highly thankful to the Animal House staff (Raj Kumar and Ankit) of AllMS, Bhopal for their immense help and support.

\section{Conflicts of interest: Nil}

Funding: Nil 


\section{References}

1. Robbins and cotran. Inflammation and repair. Pathologic basis of disease, 10th edition, 2020:71-113.

2. Bindu S, Mazumder S, Bandyopadhyay U. Non-steroidal anti-inflammatory drugs (NSAIDs) and organ damage: A current perspective.Biochem Pharmacol.2020 10;180:114147. doi: 10.1016/j.bcp.2020.114147.

3. National Center for Biotechnology Information (2020). PubChem Compound Summary for CID 5720, Zaltoprofen; Retrieved September 18, 2020 from https://pubchem.ncbi.nlm.nih.gov/compound/Zaltoprofen.

4. Tsurumi K, Kyuki K, Niwa M, Mibu H, Fujimura H. Pharmacological investigations of the new anti-inflammatory agent 2-(10,11-dihydro-10-oxodibenzo[b,f] thiepin-2-yl) propionic acid. 1st communication: inhibitory effects of rat paw edema. Arzneim- Forsch/Drug Res 1986;36:1801-5.

5. Santenna C, Kumar S, Balakrishnan S, Jhaj R, Ahmed SN. A comparative experimental study of analgesic activity of a novel non-steroidal antiinflammatory molecule - zaltoprofen, and a standard drug- piroxicam, using murine models. J Exp Pharmacol. 2019;11:85-91.

6. Kameyama, T., Nabeshima, T., Yamada, S., Sato, M. Analgesic and antiinflammatory effects of 2-(10,11-dihydro-10-oxodibenzo[b, f]thiepin2-yl) propionic acid in rat and mouse. Arzneim- Forsch/Drug Res 1987: 19-26.

7. Hirate K, Uchida A, Ogawa Y, Arai T, Yoda K. Zaltoprofen, a non-steroidal anti-inflammatory drug, inhibits bradykinin-induced pain responses without blocking bradykinin receptors. Neurosci Res. 2006: 288-94.

8. Kohno, Tatsuro. Zaltoprofen Inhibits Bradykinin-Mediated Enhancement of Glutamate Receptor Activity in Substantia Gelatinosa Neurons, Anesthesia \& Analgesia 2011:412-6.

9. Tang HB, Inoue A, Oshita K, Hirate K, Nakata Y. Zaltoprofen inhibits bradykinin-induced responses by blocking the activation of second messenger signaling cascades in rat dorsal root ganglion cells. Neuropharmacology 2005:1035-42.

10. Matsumoto M, Inoue M, Ueda H. NSAID zaltoprofen possesses novel anti-nociceptive mechanism through blockage of B2-type bradykinin receptor in nerve endings. Neurosci Lett 2006:249-53.

11. Hongxia Cui, Peng Quan, Zhuang Zhou, Liang Fang. Development of a drug-in-adhesive patch combining ion pair and chemical enhancer strategy for transdermal delivery of zaltoprofen: pharmacokinetic, pharmacodynamic and in vitro/ in vivo correlation evaluation. Drug Delivery 2016.23:3461-70.

12. Higuchi T, Takeuchi A, Munesue S, Yamamoto N, Hayashi K, Kimura H, et al. Anti-tumor effects of a nonsteroidal anti-inflammatory drug zaltoprofen on chondrosarcoma via activating peroxisome proliferator-activated receptor gamma and suppressing matrix metalloproteinase-2 expression. Cancer Med. 2018; 7:1944-54.

13. Takeuchi A, Nomura A, Yamamoto N, Hayashi K, Igarashi K, Tandai S, et al. Randomized placebo-controlled double-blind phase II study of zaltoprofen for patients with diffuse-type and unresectable localized tenosynovial giant cell tumors: a study protocol. BMC Musculoskelet Disord. 2019; 20:68.

14. Okamoto T, Masuda Y, Kawasaki T and Okabe S. Zaltoprofen prevents carbon tetrachloride-induced reduction of body weight in rats. Int $\mathrm{J}$ Mol Med 2001.7: 101-4.

15. Patil K.R., Patil C.R. Anti-inflammatory activity of bartogenic acid containing fraction of fruits of Barringtonia racemosa Roxb in acute and chronic animal models of inflammation. J.Tradit.Complementary Med. 2017:86-93.

16. Wheeler-Aceto, H., Cowan, A. Neurogenic and tissue-mediated components of formalin-induced edema: Evidence for supraspinal regulation. Agents and Actions 1991.34: 264-9.

17. Juma K.M., Ahmed Z.A., Numan I.T., Hussain S A R.Dose-dependent anti-inflammatory effect of silymarin in experimental animal model of chronic inflammation. Afr. J. Pharm. Pharmacol. 2009; 3:242-7.

\section{Tables}

\begin{tabular}{l|l|l|l|l|l|}
\hline Inimal Group & Before Drug & 0 Hours & $\mathbf{1}$ hour & 2 hour & hour \\
\hline :ontrol (NS 10ml/kg, PO) & $1.11 \pm 0.114$ & $1.61 \pm 0.074$ & $1.71 \pm 0.067$ & $1.94 \pm 0.101$ & $2.31 \pm 0.348$ \\
\hline :LT10(10 mg/kg, PO) & $1.08 \pm 0.147$ & $1.58 \pm 0.057$ & $1.59 \pm 0.048$ & $1.48 \pm 0.062$ & $1.43 \pm 0.068^{* * *}$ \\
\hline :LT20(20 mg/kg, PO) & $1.10 \pm 0.116$ & $1.60 \pm 0.193$ & $1.55 \pm 0.189^{*}$ & $1.45 \pm 0.132^{* * *}$ & $1.36 \pm 0.129^{* * * *}$ \\
\hline 'RC10(10 mg/kg, PO) & $1.00 \pm 0.031$ & $1.58 \pm 0.092$ & $1.48 \pm 0.083^{* *}$ & $1.40 \pm 0.067^{* * *}$ & $1.28 \pm 0.088^{* * *}$ \\
\hline
\end{tabular}

Table I : Effect of Different Drugs Dosages On Paw Volume In Carrageenan-Induced Paw Oedema in Rats. Values are expressed as mean \pm Standard Deviation ( $n=6$ in each group).

$* * * \mathrm{P}<0.001, * * \mathrm{P}<0.01, * \mathrm{P}<0.05$ when compared to control.

Abbreviations: NS: Normal saline; ZLT10: Zaltoprofen 10 mg/kg, ZLT20: Zaltoprofen 20 mg/kg, PRC10: Piroxicam 10 mg/kg, PO: Per Os. 


\begin{tabular}{l|c|c|c|c|c|}
\hline Animal Group & Before Drug & 0 Hours & \%inhibition at 1 hour & \%inhibition at 2 hour. & \%inhibition at 3 hour. \\
\hline Control (NS 10 ml/kg PO) & 0.00 & 0.00 & 0.00 & 0.00 & 0.00 \\
\hline ZLT(10 mg/kg PO) & 2.69 & 2.07 & 7.12 & 24.10 & 38.24 \\
\hline ZLT(20 mg/kg PO) & 1.35 & 0.83 & 9.26 & 25.21 & 41.05 \\
\hline PRC(10 mg/kg PO) & 10.03 & 1.86 & 13.55 & 27.96 & 44.52 \\
\hline
\end{tabular}

Table II: Effect of Different Drug Dosages on Percentage Inhibition Of Carrageenan Induced Acute Inflammation (n=6 in each group).

Abbreviations: NS: Normal Saline; ZLT10: Zaltoprofen 10 mg/kg, ZLT20: Zaltoprofen 20 mg/kg, PRC10: Piroxicam 10 mg/kg, PO: Per Os.

\begin{tabular}{|c|c|c|c|c|c|c|c|c|c|c|}
\hline $\begin{array}{l}\text { nal } \\
\text { up }\end{array}$ & Day 0 & Day 1 & Day 2 & Day 3 & Day 4 & Day 5 & Day 6 & Day 7 & Day 8 & $\overline{\text { Day }}$ \\
\hline $\begin{array}{l}\text { trol } \\
\mathrm{Cl} \\
\mathrm{l} / \mathrm{kg} \\
\mathrm{l} \\
\end{array}$ & $3.45 \pm 0.207$ & $6.78 \pm 0.331$ & $7.58 \pm 0.440$ & $7.12 \pm 0.488$ & $6.18 \pm 0.360$ & $5.70 \pm 0.276$ & $5.43 \pm 0726$ & $5.15 \pm 0.616$ & $4.93 \pm 0.484$ & 4.73 \\
\hline $\begin{array}{l}10(10 \\
\mathrm{kg} \mathrm{P}\end{array}$ & $3.38 \pm 0.223$ & $6.37 \pm 0.378$ & $6.15 \pm 0.513$ & $5.93 \pm 0.308^{* *}$ & $5.62 \pm 0.214$ & $5.35 \pm 0.459$ & $5.20 \pm 0.452$ & $4.92 \pm 0.519$ & $4.75 \pm 0.362$ & 4.60 \\
\hline $\begin{array}{l}120 \\
\mathrm{~kg} \mathrm{P}\end{array}$ & $3.30 \pm 0.167$ & $4.90 \pm 0.576$ & $5.87 \pm 0.501$ & $5.42 \pm 0.512^{* * *}$ & $5.15 \pm 0.327$ & $4.90 \pm 0.424$ & $4.77 \pm 0.423$ & $4.62 \pm 0.387$ & $4.52 \pm 0.387^{* *}$ & 4.40 \\
\hline $\begin{array}{l}(10 \\
\mathrm{kg}\end{array}$ & $3.05 \pm 0.259$ & $4.55 \pm 0.622$ & $4.90 \pm 0.420$ & $4.58 \pm 0.331$ & $4.42 \pm 0.382$ & $4.35 \pm 0.383^{* *}$ & $4.32 \pm 0.172$ & $4.15 \pm 0.164$ & $4.02 \pm 0.402$ & 3.87 \\
\hline
\end{tabular}

Table 3 : Effect of Different Drug Dosages On Formalin Induced Chronic Inflammation in Rats. Values are expressed as Mean \pm Standard Deviation ( $n=6$ in each group).

$* * * \mathrm{P}<0.001, * * \mathrm{P}<0.01, * \mathrm{P}<0.05$ when compared to control.

Abbreviations: NS: Normal saline; ZLT10: Zaltoprofen 10 mg/kg, ZLT20: Zaltoprofen 20 mg/kg, PRC10: Piroxicam 10 mg/kg, PO: Per Os.

\begin{tabular}{l|l|l|}
\hline Animal Group & Mean difference in LCS (mm) & Percentage Inhibition \\
\hline Control ( NS 10ml/kg) & $1.23 \pm 0.027$ & - \\
\hline ZLT10 (10 mg/kg P O) & $1.17 \pm 0.063^{* * *}$ & 18.90 \\
\hline ZLT20 (20 mg/kg P O) & $1.05 \pm 0.350^{* * *}$ & 27.76 \\
\hline PRC10 (10 mg/kg P O) & $0.48 \pm 0.049^{* * *}$ & 35.60 \\
\hline
\end{tabular}

Table 4: Effect of Different Drugs Doses On The Linear Cross-Section (LCS) Below The Ankle Joint In The Formalin-Induced Chronic Inflammation Or Arthritis In Rats. Values are expressed as Mean \pm Standard Deviation ( $\mathrm{n}=6$ in each group). $* * * \mathrm{P}<0.001$ when compared to control.

Abbreviations: NS: Normal saline 10 ml/kg; ZLT10: Zaltoprofen 10 mg/kg, ZLT20: Zaltoprofen 20 mg/kg, PRC10: Piroxicam 10 mg/kg, PO: Per Os.

\section{Figures}




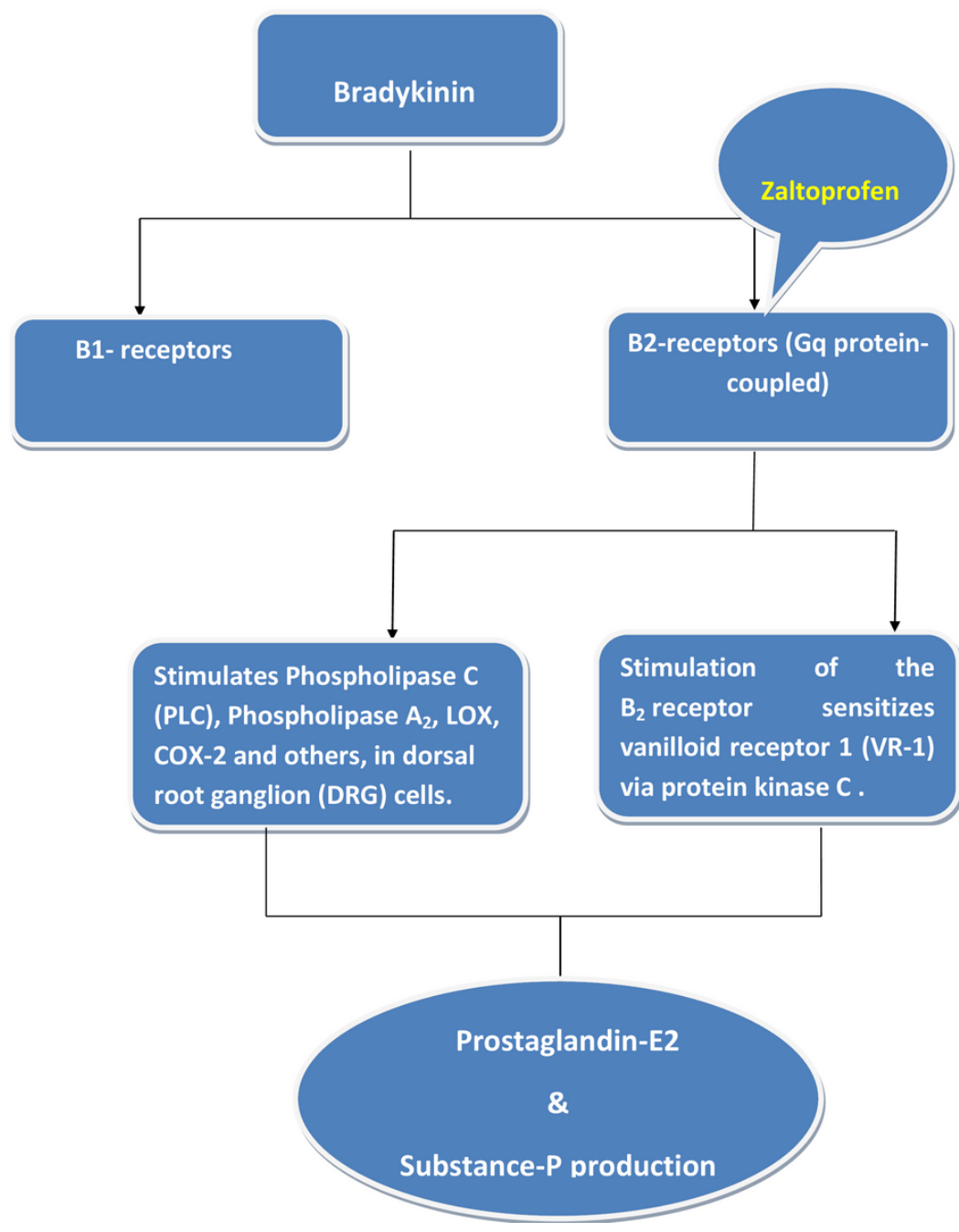

\section{Figure 1}

Mechanism of Action of Zaltoprofen.

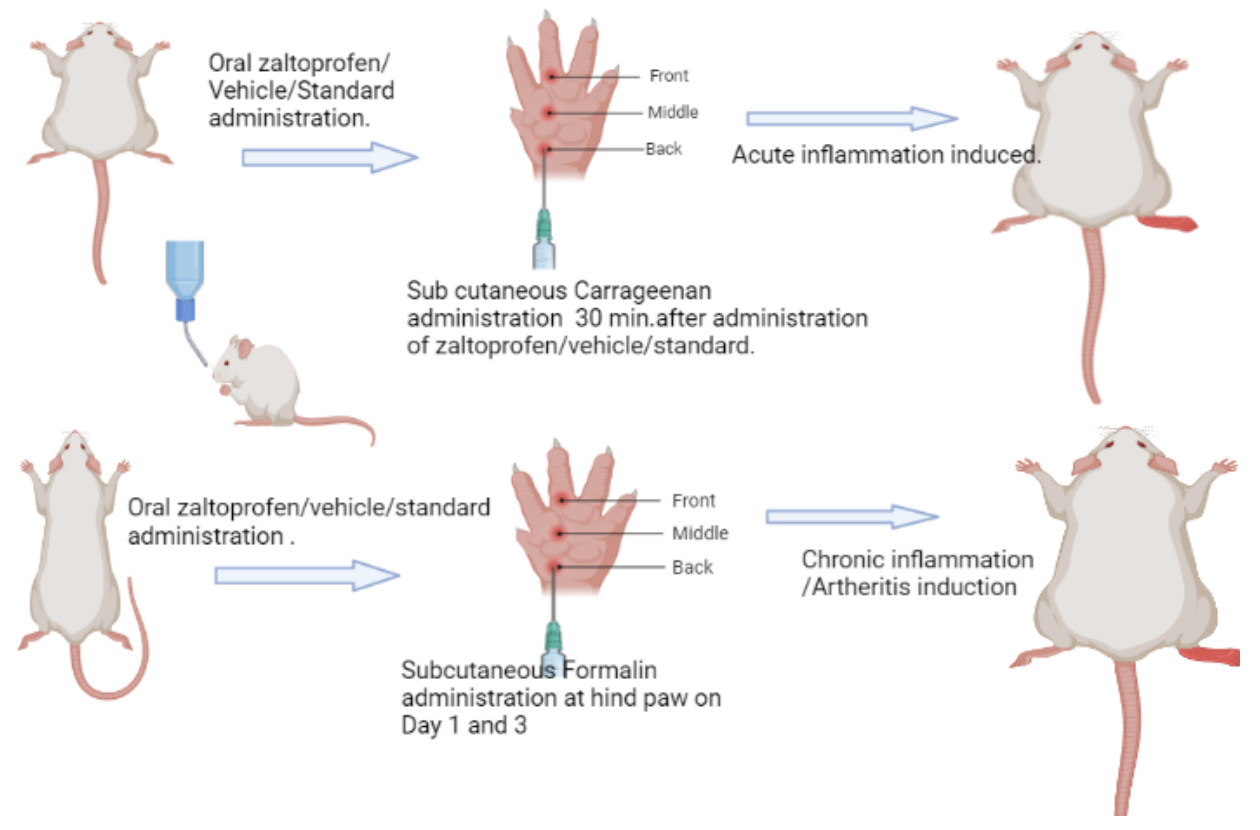

Figure 2

Graphical abstract of Study methodology. 


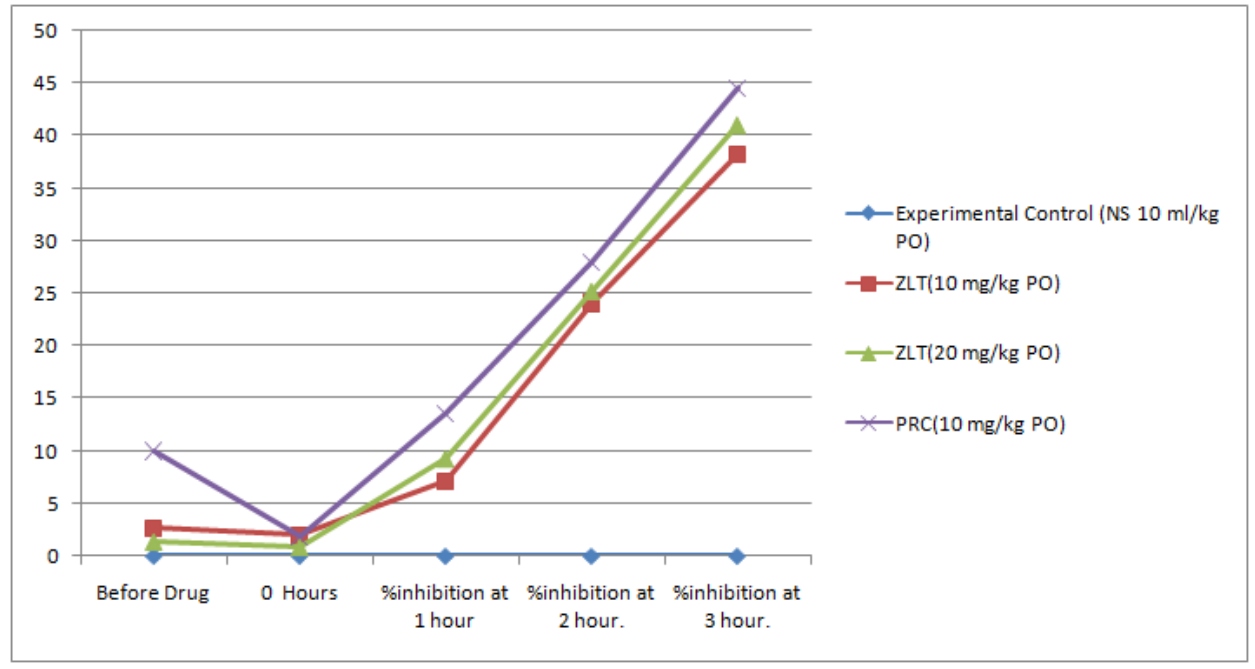

Figure 3

Effect of different drugs on Carrageenan induced paw oedema at different time intervals in Rats.

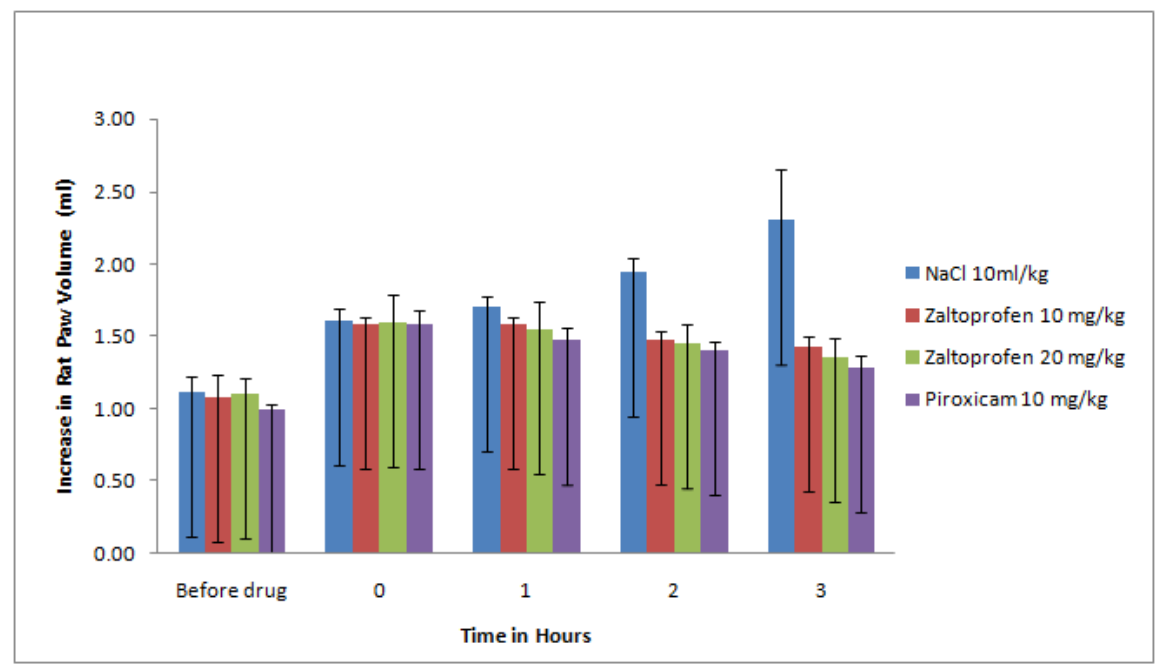

Figure 4

Effect of different drug dosages on percentage inhibition (mean) of Carrageenan-induced acute inflammation in Rats.

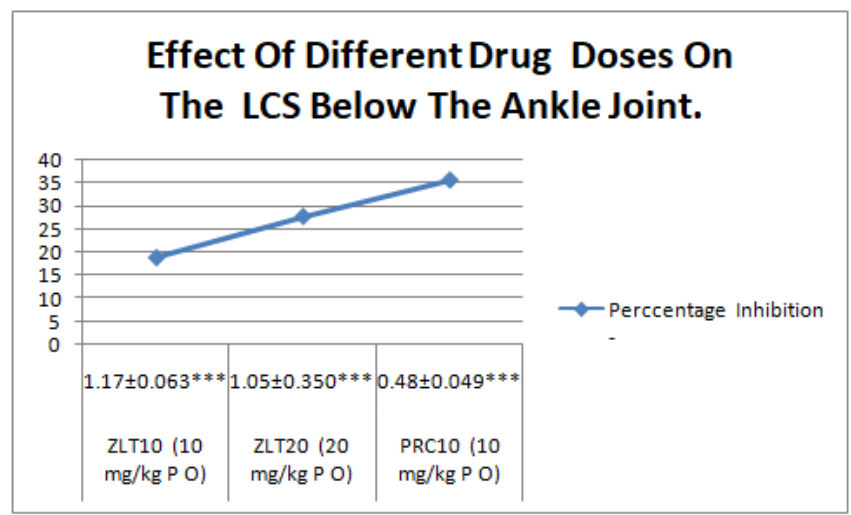

Figure 5

Effect of different drugs on Formalin-induced chronic inflammation or arthritis in Rats. 